\title{
Situação do aleitamento materno em duas capitais brasileiras: uma análise comparada
}

\author{
Breastfeeding in two Brazilian State capitals: \\ a comparative analysis
}

Pedro Makumbundu Kitoko ${ }^{1}$

Marina Ferreira Réa ${ }^{2}$

Sonia Isoyama Venancio 2

Ana Cláudia Cavalcanti Peixoto de Vasconcelos 3

Evanguelia Kotzias Atherino dos Santos 4

Carlos Augusto Monteiro 1

\footnotetext{
1 Núcleo de Pesquisas Epidemiológicas em Nutrição e Saúde, Universidade de São Paulo. Av. Dr. Arnaldo 715, São Paulo, SP 01246-904, Brasil. carlosam@usp.br 2 Núcleo de Investigação em Saúde da Mulher e da Criança, Instituto de Saúde, Secretaria Estadual de Saúde de São Paulo.

Rua Santo Antônio 590, 2o andar, São Paulo, SP 01314-000, Brasil. marifrea@usp.br 3 Departamento de Nutrição, Universidade Federal da Paraíba. Cidade Universitária, Campus 1, João Pessoa, PB 58059-900, Brasil. 4 Maternidade Carmela Dutra, Departamento de Enfermagem, Universidade Federal de Santa Catarina. Rua Irmã Benwarda 208, Florianópolis, SC 88015-270, Brasil.
}

\begin{abstract}
This paper describes rapid assessment of infant feeding practices based on surveys conducted on National Immunization Day in the cities of Florianópolis and João Pessoa, Brazil. Two different infant feeding patterns emerge clearly in the data analysis. Most infants begin breastfeeding, but exclusive breastfeeding (EBF) from 0-4 months (46.3\% in Florianópolis and 23.9\% in João Pessoa) and timely complementary feeding rates (32.2\% in Florianópolis and $24.8 \%$ in João Pessoa) are below recommended standards. EBF and breastfeeding duration medians were 53 and 238 days, respectively, in Florianópolis and 16.5 and 195 days, respectively, in João Pessoa. The results pointed to increasing breastfeeding rates and duration medians in Florianópolis as compared to João Pessoa. Use of these data could improve planning and monitoring of breastfeeding activities and infant nutrition policies.
\end{abstract}

Key words Breast Feeding; Supplementary Feeding; Feed; Statistical Analysis

Resumo Este artigo descreve a análise dos resultados de diagnóstico rápido das práticas de alimentação infantil valendo-se de inquéritos realizados em amostras de crianças menores de um ano em Dias Nacionais de Vacinação, nas cidades de Florianópolis e João Pessoa. A análise de dados sugere existência de processos diferenciados dessas práticas nas cidades estudadas. A maioria das crianças inicia a amamentação, mas a prevalência do aleitamento materno exclusivo (AME) em menores de quatro meses de idade (46,3\% em Florianópolis e 23,9\% em João Pessoa) e de alimentação complementar oportuna (32,2\% em Florianópolis e 24,8\% em João Pessoa) encontram-se aquém das metas preconizadas. As medianas de duração de AME e de amamentação foram de 53 e 238 dias, respectivamente, em Florianópolis e, 16,5 e 195 dias, respectivamente, em João Pessoa. A situação é melhor em Florianópolis quando comparado com João Pessoa. Espera-se que esses resultados sirvam de base para monitorar a evolução dos indicadores e planejar ou redirecionar as atividades pró-amamentação e as políticas nutricionais.

Palavras-chave Aleitamento Materno; Suplementação Alimentar; Alimentação; Análise Estatística 


\section{Introdução}

A amamentação exclusiva nos primeiros quatro a seis meses de vida e a manutenção da amamentação, complementada por outros alimentos, dos seis aos vinte quatro meses de idade ou mais, constituem práticas indispensáveis para a saúde e o desenvolvimento da criança, assim como para a saúde da mãe (Victora et al., 1987; WHO/UNICEF/USAID/SIDA, 1990). Partindo desse preceito, as autoridades sanitárias do Brasil vêm desenvolvendo, desde 1981, um conjunto de atividades pró-amamentação coordenadas pelo Ministério da Saúde. A recuperação da prática de amamentar, após décadas de declínio, tem sido em parte atribuída a esses esforços (Rea \& Berquó, 1995).

Estudos de âmbito nacional revelaram um aumento substancial da duração mediana da amamentação no país, que passou de 2,5, em 1975, para 5,5 meses, em 1989 (Venancio \& Monteiro, 1998). Os resultados da Pesquisa Nacional sobre Demografia e Saúde (PNDS), realizada em 1996, mostram que a tendência de aumento se mantém, estimando a duração mediana da amamentação por volta de sete meses (BEMFAM, 1997; Monteiro, 1997); no entanto, a prevalência de amamentação exclusiva em crianças menores de quatro meses de idade, estimada em $40 \%$, ainda é muito baixa, muito distante de universalização dessa prática preconizada pela Organização Mundial de Saúde (OMS) e pelo Fundo das Nações Unidas para a Infância (UNICEF) (WHO, 1994). O uso da mamadeira e a introdução de água e chás continuam sendo constatados em diferentes estudos, sendo o Brasil um dos países da América Latina com menor prevalência da amamentação exclusiva nos primeiros meses de vida (Boerma et al., 1991; Pérez-Escamilla, 1993).

Diante desse quadro, e tendo em vista os conhecidos benefícios da amamentação, justifica-se a necessidade de se implantar no país um sistema de avaliação que permita o diagnóstico rápido das práticas de alimentação no primeiro ano de vida. Sistemas apoiados em levantamentos periódicos de padrões de alimentação infantil em Dias Nacionais de Vacinação (DNV), por sua praticidade, baixo custo e confiabilidade, caso seja alta a cobertura populacional da campanha, afiguram-se como promissores. Com a proposta de descentralização do Sistema Único de Saúde (SUS), cabe aos municípios a responsabilidade pelo planejamento, execução e avaliação das ações de saúde, levando em conta as diretrizes e políticas nacionais. Assim sendo, é fundamental conhecer os indicadores de saúde da população, dentre os quais os de alimentação infantil.
O presente artigo pretende analisar os resultados de diagnósticos rápidos das práticas de alimentação infantil obtidos com base em inquéritos realizados em amostras de crianças menores de um ano em DNV, nas cidades de Florianópolis e João Pessoa. As duas cidades foram selecionadas devido ao interesse que profissionais e autoridades de saúde dessas localidades mostraram em conhecer a situação local do aleitamento materno, com vistas ao planejamento ou redirecionamento de ações que visem melhorar os níveis de indicadores de alimentação infantil como parte do processo de desenvolvimento sanitário. Vale lembrar que as duas cidades pertencem a dois pólos opostos no que se refere à distribuição regional de indicadores do desenvolvimento social e econômico. João Pessoa é a capital do Estado de Paraíba, um dos nove Estados da Região Nordeste, considerada a mais pobre do país. Florianópolis é a capital do Estado de Santa Catarina, localizado na Região Sul, que forma com a Região Sudeste a área mais desenvolvida do Brasil (De Sousa, 1992; Da Silva-Simões \& Monteiro, 1995; Lavinas \& Magina, 1996).

\section{Material e métodos}

A coleta de dados foi realizada no dia 16 de agosto de 1997, DNV, sendo parte do projeto conjunto do Núcleo de Pesquisas Epidemiológicas em Nutrição e Saúde da Universidade de São Paulo (NUPENS/USP) e do Núcleo de Investigação em Saúde da Mulher e da Criança, Instituto de Saúde, Secretaria Estadual de Saúde de São Paulo (NISMC/IS/SES-SP), denominado "Avaliação de Práticas Alimentares no Primeiro Ano de Vida em Dias Nacionais de Vacinação". Tomando-se por base uma amostragem probabilística, foram sorteadas crianças menores de um ano, que compareceram nos trinta postos de vacinação, na cidade de João Pessoa e em um terço dos 92 postos existentes, na cidade de Florianópolis.

\section{Amostragem}

Nas duas cidades planejou-se obter uma amostra de pelo menos mil crianças com idade entre 0 e 11 meses. Esse contigente de crianças seria suficiente para investigar, com erro de $10 \%$, a prevalência de crianças menores de quatro meses em amamentação exclusiva. Em João Pessoa, aplicou-se um processo de amostragem por conglomerados em estágio único, e o coeficiente de variação no número médio de crianças por conglomerado (posto de vacina- 
ção) não excedeu 15\%. O plano de amostragem baseou-se no mapa de vacinação do ano anterior. Em Florianópolis, a amostra foi definida por sorteio aleatório de um terço de todos os postos de vacinação já que o município não dispunha dos dados relativos ao número de crianças menores de um ano vacinadas em cada posto no ano anterior à pesquisa.

\section{Coleta dos dados}

A todos os acompanhantes das crianças menores de um ano da amostra foi aplicado um questionário com perguntas sobre todos os alimentos consumidos nas últimas 24 horas, incluindo leite materno, outros leites e alimentos em geral como chás e água. Quando a criança foi acompanhada por sua mãe, incluíram-se dados sobre sua idade, escolaridade e ocupação. Esse questionário é suficientemente simples para ser aplicado por pessoal não especializado, após treinamento adequado. A duração média de sua aplicação, estimada em pré-testes, ficou por volta dos quatro minutos.

Em João Pessoa, os entrevistadores foram recrutados entre os estudantes do curso de nutrição, enquanto que em Florianópolis, as entrevistas ficaram a cargo de alunos do curso técnico de enfermagem e de alguns enfermeiros. Todos os entrevistadores receberam um treinamento para aplicação do questionário. Em todos os postos sorteados, entrevistaramse os acompanhantes de crianças menores de um ano, usando-se nas duas cidades o mesmo protocolo. O treinamento e a supervisão dos entrevistadores ficaram a cargo de dois autores (A.C.C.P.V. e E.K.A.S.). Adicionalmente, cada entrevistador recebeu durante o treinamento um manual que esclarecia detalhadamente as perguntas e todos os procedimentos do campo. Após o seu preenchimento, os questionários foram entregues aos supervisores.

\section{Análise dos dados}

Uma vez recebido, o material coletado em campo foi conferido pelas supervisoras. Posteriormente, procedeu-se à digitação dos dados e à criação do banco no software Epi Info 6.02 (Dean et al., 1994). Um exame de consistência foi previamente realizado. Os questionários contendo alguma inconsistência que não pôde ser solucionada e as crianças de um ano completo ou mais foram excluídos do estudo. Deste modo, dois questionários preenchidos, em João Pessoa ( $0,2 \%$ do total) e 65 , em Florianópolis $(6,2 \%$ do total), foram excluídos do estudo devido à existência de uma inconsistência grave, de informações incompletas ou à idade da criança igual ou maior que um ano.

Segundo recomendações da OMS (OPS/ OMS, 1991; UNICEF/SSB, 1995; WHO, 1997), internacionalmente aceitas, a prevalência total de amamentação (breastfeeding) em uma população corresponde à proporção de crianças que se alimentam com leite materno independentemente de consumo de outros líquidos ou alimentos sólidos/semi-sólidos. Na ausência de consumo destes, a amamentação é classificada como exclusiva (exclusive breastfeeding), podendo a criança, neste particular, ingerir além do leite materno somente suplementos minerais/vitamínicos ou medicamentos. No cálculo da proporção combinada de crianças em amamentação exclusiva e predominante (predominant breastfeeding), juntam-se às crianças exclusivamente amamentadas as que recebem, além do leite materno apenas água, chás ou sucos de frutas, sais de reidratação oral, suplementos minerais/vitamínicos, medicamentos ou, em pequena quantidade, fluidos rituais. Essa modalidade combinada é denominada amamentação plena ou completa (full breastfeeding). A amamentação é dita complementada quando a criança amamentada recebe alimento sólido/semi-sólido, independentemente de estar ou não recebendo outro leite que não o materno e outros líquidos. A OMS propõe, ainda, que se investigue na população a proporção de crianças que recebem qualquer líquido ou alimento através de mamadeiras.

Em relação à amamentação plena, os participantes da reunião da Rede Interagencial de Informações para Saúde (RIPSA) organizada pela Organização Pan-Americana de Saúde (OPAS) e o Ministério da Saúde do Brasil, em abril de 1999, não recomendaram o uso dessa expressão. Nessa reunião que contou com a participação de dois autores (M. F. R. e S. I. V.), após ter constatado que a expressão "amamentação plena ou completa” não permite entender, pelo menos em português, o que se pretende com o indicador correspondente, foi recomendado o uso de termo "amamentação exclusiva e predominante”, que foi adotado nesse artigo.

Conforme recomendação da OMS, a freqüência de amamentação exclusiva e predominante foi estimada para crianças entre zero e quatro meses de idade. A freqüência da amamentação complementada foi estimada em crianças entre seis e nove meses de idade e a de amamentação e de uso de mamadeira, em crianças entre 0 e 12 meses de idade. As estimativas e respectivos intervalos de confiança de 95\% foram calculados com emprego do programa CSAMPLE do software Epi Info 6.02 (Dean 
et al., 1994), considerando-se o posto de vacinação como unidade primária de amostragem. Esse programa permite levar em conta o delineamento amostral por conglomerados, que implica maior variância das estimativas, quando comparado com a amostragem aleatória simples. O significado estatístico das diferenças de todos os parâmetros de interesse foi avaliado pelos intervalos de confiança (Gardner \& Altman, 1986; Duffau, 1997).

Curvas que caracterizam a evolução da freqüência de diferentes modalidades da amamentação ao longo das idades, bem como a duração mediana da mesma foram obtidas pela análise de regressão logística. Esta última permite estimar a probabilidade $\mathrm{P}(\mathrm{Y}=1)$ de amamentação em determinada idade, que é uma função logística: $P(Y=1)=1 / 1+\exp (-\propto-\beta x)$, onde $\mu$ e b são coeficientes de regressão e $\mathrm{x}=$ idade. A duração mediana corresponde ao ponto de inflexão Xm da curva, de modo que $\mathrm{Xm}=-\propto / \beta$, sendo $\mathrm{P}(\mathrm{Y}=1)=50 \%$ (Silva-Ayçaguer, 1995). Fez-se uso de programa SPSS 6.0 (Norusis, 1993) para realizar a análise de regressão logística.

\section{Resultados}

Na cidade de Florianópolis, que conta com cerca de 6.327 crianças menores de um ano, a meta estabelecida em 1997 foi alcançada durante a Campanha de Vacinação. Em João Pessoa, que referia 10.161 crianças da mesma faixa etária, a cobertura vacinal não ultrapassou $80 \%$. Foi registrada maior concentração de crianças em Florianópolis, com uma média de 30 por posto contra 23, em João Pessoa. A pesquisa envolveu 950 crianças menores de um ano nesta última cidade e 990, em Florianópolis. As crianças foram acompanhadas por suas mães em 87,5\% dos casos, em João Pessoa e 86,9\% dos casos, em Florianópolis. A idade média das crianças estudadas foi semelhante em ambas as cidades $(190,2 \pm 101,8$ dias, em João Pessoa e, 186,3 \pm 101,7 dias, em Florianópolis).

A Tabela 1 apresenta a distribuição da amostra de crianças segundo quatro características maternas: idade, paridade, escolaridade e ocupação. Observa-se que as mães de João Pessoa, quando comparadas às de Florianópolis, são mais jovens, têm menor nível de escolaridade e trabalham menos fora de casa. As Figuras 1 e 2 reproduzem as curvas da prevalência de amamentação construídas pela regressão logística e retratam padrões de diferentes modalidades de amamentação observadas em ambas as cidades. Apesar da universalidade de iniciação da amamentação, verifica-se, em todas as curvas, um declive que inicia logo nos primeiros dias de vida, sendo mais acentuado em João Pessoa do que em Florianópolis, particularmente em relação à amamentação exclusiva e à modalidade de amamentação exclusiva e predominante. Esse fato evidencia a importância do desmame precoce nas cidades estudadas.

A Tabela 2 reproduz a prevalência de diferentes modalidades de alimentação infantil em cada uma das cidades. Observa-se que João Pessoa supera Florianópolis apenas no uso da mamadeira, que é um indicador ruim. Com exceção do indicador combinado (amamentação exclusiva + predominante), não se observa superposição de intervalos de confiança dos indicadores entre as duas cidades, sugerindo diferenças estatisticamente significantes.

Vale também referir que as proporções de crianças que usaram mamadeiras, $61,4 \%$, em Florianópolis e 77,6\%, em João Pessoa, praticamente correspondem às freqüências de consumo de leite artificial (62,6\%, em Florianópolis e $77,1 \%$, em João Pessoa). Essa coincidência sugere que a mamadeira tem sida usada para oferecer leite artificial às crianças. Na maioria das vezes, o leite (integral, fresco, pasteurizado etc.) oferecido às crianças não é modificado para a alimentação de lactentes, observando-se 12,6\% de consumo de fórmula infantil, em João Pessoa, e o dobro dessa proporção, em Florianópolis. A diferença no tipo de leite artificial consumido pode estar associada às condições econômicas experimentadas pelas famílias. Sabese que o padrão de desmame das crianças, além de influir no estado nutricional destas, pode espelhar as condições econômicas vividas pelas populações.

Apenas 32,2\% das crianças amamentadas de Florianópolis e $24,8 \%$ de João Pessoa receberam alimento semi-sólido/sólido numa faixa etária (6 a 9 meses) em que a introdução de alimentação complementar é considerada oportuna (WHO, 1997). Observou-se também que 40,5\% das crianças de João Pessoa, de 6 a 9 meses, que receberam alimentação complementar, não comeram feijão nem carne, enquanto que, em Florianópolis, 83,5\% das crianças nessa condição, consumiram estas duas importantes fontes de ferro. O menor nível de consumo de feijão e de carne não somente pode estar associado ao risco de anemia ferropriva na comunidade mas também pode expressar menor nível de desenvolvimento econômico desta.

A duração mediana das principais categorias de amamentação estão apresentadas na Tabela 3. O diferencial de duração mediana de amamentação entre ambas as cidades é favo- 
rável a Florianópolis. A relação deste indicador entre as cidades (Florianópolis/João Pessoa), no que se refere à amamentação exclusiva, mostra que a prevalência é 3,2 vezes maior em Florianópolis quando comparada à observada em João Pessoa. Nas outras modalidades, a magnitude da relação é menor, sendo mais baixa com relação à amamentação $(1,2)$ e intermediária, quando a modalidade é amamentação exclusiva e predominante $(1,8)$.

\section{Comentários e conclusões}

A metodologia proposta, coletando dados sobre alimentação infantil em DNV, apresenta inúmeras vantagens como o seu baixo custo, a relativa facilidade no seu planejamento e o alto grau de adesão da população. A estratégia adotada pode facilitar o monitoramento da evolução de indicadores selecionados e a comparabilidade dos resultados. O método de current status usado possibilita minimizar o recall bias que assume uma grande importância nos estudos retrospectivos. Os indicadores da prática de amamentação propostos pela OMS/UNICEF (OPS/OMS, 1991; WHO, 1997) mostraram-se passíveis de coleta adequada, quando parte de um questionário elaborado com cuidado. Na aplicação do protocolo, não houve relatos, por parte das equipes das duas cidades, de dificuldades para a realização da pesquisa durante a campanha de vacinação.

No entanto, a qualidade dos resultados pode ser questionada, quando a cobertura da campanha de vacinação é baixa, havendo menor comparecimento de crianças em relação ao número esperado. Com a ampla mobilização de instituições e da população no Brasil, esta cobertura costuma alcançar $90 \%$ ou mais das metas propostas (Silva et al., 1991). A relativa baixa cobertura registrada em João Pessoa originou uma proporção de $20 \%$ de perdas de crianças. A caracterização destas foi impossibilitada por não existir mecanismo de identificálas de forma rápida. A opção pela amostragem por conglomerados acaba, de qualquer forma, por dar força à idéia de que, em condição de cobertura vacinal de pelo menos $80 \%$ da população-alvo, é possível que as amostras trabalhadas sejam representativas.

Conforme Barros \& Victora (1991), por ser muito mais prática, é comum utilizar-se a amostragem por conglomerados. Esse método é muito mais rápido e menos dispendioso do que a escolha de crianças em todos os postos (Nunes-da-Silva, 1998). Nos três últimos anos, tem-se verificado no Brasil uma tendência cres-
Tabela 1

Distribuição de crianças segundo características maternas. João Pessoa

e Florianópolis, 1997.

\begin{tabular}{|c|c|c|c|c|}
\hline \multirow[t]{2}{*}{ Características maternas } & \multicolumn{2}{|c|}{ João Pessoa } & \multicolumn{2}{|c|}{ Florianópolis } \\
\hline & amostra & $\%$ & amostra & $\%$ \\
\hline \multicolumn{5}{|l|}{ Idade materna (anos) } \\
\hline$<20$ & 187 & 22,5 & 130 & 15,1 \\
\hline 20 a 24 & 298 & 35,9 & 255 & 29,7 \\
\hline 25 a 29 & 205 & 24,7 & 212 & 24,7 \\
\hline 30 a 34 & 80 & 9,6 & 177 & 20,6 \\
\hline$\geq 35$ & 61 & 7,3 & 86 & 10,0 \\
\hline \multicolumn{5}{|l|}{ Paridade } \\
\hline Primípara & 398 & 48,1 & 423 & 49,2 \\
\hline Multípara & 430 & 51,9 & 436 & 50,8 \\
\hline \multicolumn{5}{|l|}{ Anos de escolaridade materna } \\
\hline 0 & 60 & 7,3 & 24 & 2,8 \\
\hline $1-3$ & 122 & 14,8 & 60 & 7,0 \\
\hline $4-8$ & 361 & 43,7 & 343 & 39,9 \\
\hline $9-11$ & 206 & 24,9 & 317 & 36,9 \\
\hline$\geq 12$ & 77 & 9,3 & 115 & 13,4 \\
\hline \multicolumn{5}{|l|}{ Ocupação materna } \\
\hline Trabalho doméstico próprio & 482 & 60,4 & 377 & 45,8 \\
\hline Trabalho informal & 148 & 18,5 & 166 & 20,1 \\
\hline Trabalho formal & 168 & 21,1 & 281 & 34,1 \\
\hline
\end{tabular}

Figura 1

Prevalência (\%) da amamentação no primeiro ano de vida. Florianópolis, 1997

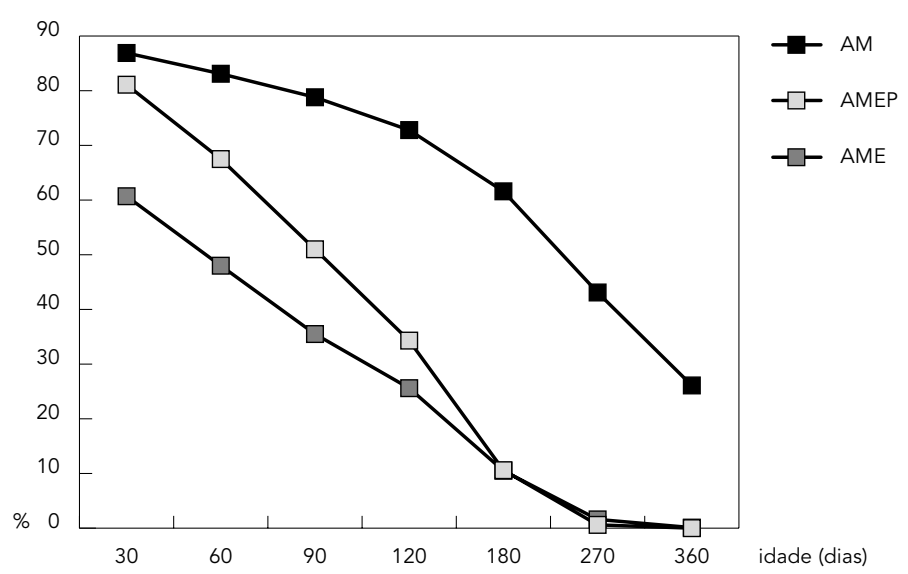

$\mathrm{AM}=$ Amamentação; $\mathrm{AME}=$ Amamentação exclusiva

$\mathrm{AMEP}=$ Amamentação exclusiva e predominante. 
cente de estudos que investigam a situação atual de amamentação nos DNV. Foi possível identificar uma dúzia de estudos que aplicaram, em diferentes cidades, a metodologia proposta neste trabalho (Parada et al., 1997; Monego et al., 1998; Vieira et al., 1998; entre outros). Mas a comparabilidade dos resultados é ainda difícil por falta de uniformidade nos indicadores empregados. Pode-se comparar apenas os resultados obtidos em cinco cidades das quais duas são da região Sudeste (Campinas e Rio de Janeiro), uma da região Centro-Oeste

Figura 2

Prevalência (\%) da amamentação no primeiro ano de vida. João Pessoa, 1997.

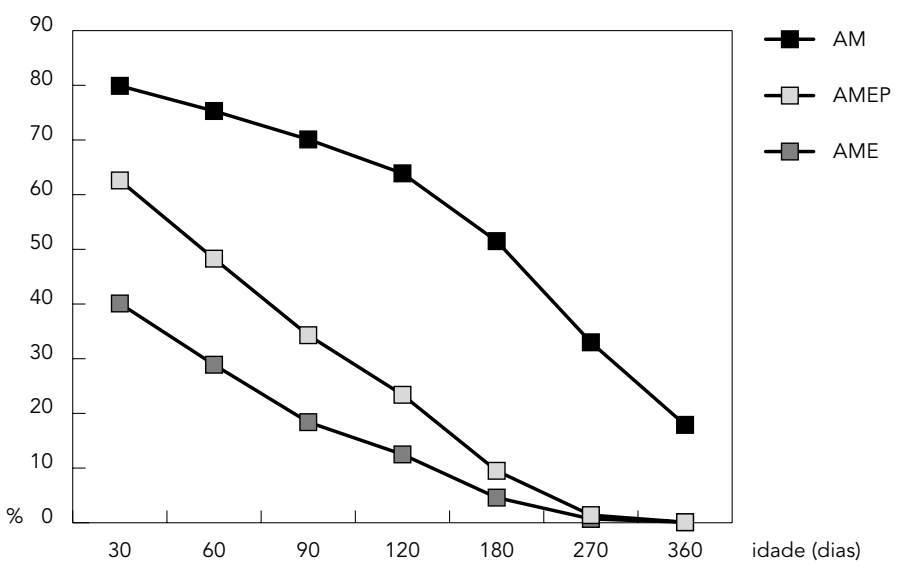

$\mathrm{AM}=$ Amamentação; $\mathrm{AME}=$ Amamentação exclusiva; AMEP $=$ Amamentação exclusiva e predominante.
(Goiânia), uma do Sul (Blumenau) e uma do Nordeste (Feira de Santana).

As cidades de Campinas e de Feira de Santana apresentaram níveis de indicadores semelhantes aos obtidos no presente trabalho, em Florianópolis, com uma duração mediana de amamentação exclusiva de sessenta dias, na primeira, e uma prevalência de amamentação exclusiva em menores de quatro meses de $46 \%$ (Vieira et al., 1998), na segunda. A cidade de Blumenau com uma duração mediana de amamentação exclusiva de trinta dias (Arcoverde et al., 1997), encontra-se numa posição intermediária. As cidades do Rio de Janeiro, com uma duração mediana de amamentação exclusiva de 13 dias (Mendes-Gomes et al., 1998) e a de Goiânia (Monego et al., 1998), onde a prevalência de amamentação exclusiva em menores de quatro meses e a duração mediana desta categoria de amamentação foram estimadas em, respectivamente, $18,5 \%$ e 12 dias. Tais percentuais e números de dias constituem, com a cidade de João Pessoa, as áreas com piores níveis dos indicadores.

Em todas as cidades onde foi aplicada metodologia semelhante a adotada no presente estudo, observou-se uma prevalência de amamentação exclusiva abaixo de $50 \%$ em menores de quatro meses e uma duração de amamentação exclusiva variando entre zero e sessenta dias. A amamentação exclusiva é interrompida muito cedo, na maioria das cidades brasileiras, com a introdução precoce de água e, sobretudo, chás. Quando comparados aos dados de pesquisas nacionais (Monteiro, 1997; Venancio \& Monteiro, 1998), esses resultados revelam que, embora exista uma situação de amamentação cada vez melhor no país, a pre-

Tabela 2

Indicadores de padrões de alimentação infantil no primeiro ano de vida. Florianópolis e João Pessoa, 1997.

\begin{tabular}{|c|c|c|c|c|c|c|}
\hline \multirow[t]{2}{*}{ Indicador } & \multicolumn{3}{|c|}{ Florianópolis } & \multicolumn{3}{|c|}{ João Pessoa } \\
\hline & $\mathrm{n}$ & $\%$ & IC $95 \%$ & $n$ & $\%$ & IC $95 \%$ \\
\hline \multicolumn{7}{|l|}{ Crianças menores de 4 meses } \\
\hline Em amamentação exclusiva & 287 & 46,3 & $37,8-54,8$ & 285 & 23,9 & $17,4-30,2$ \\
\hline Em amamentação exclusiva e predominante & 287 & 64,5 & $58,1-70,7$ & 285 & 41,1 & $34,8-47,3$ \\
\hline \multicolumn{7}{|l|}{ Crianças de 6 a 9 meses } \\
\hline Em amamentação complementada & 335 & 32,2 & $22,1-41,9$ & 314 & 24,8 & $19,2-30,3$ \\
\hline \multicolumn{7}{|l|}{ Crianças menores de 12 meses } \\
\hline Amamentadas & 990 & 59,4 & $55,3-63,5$ & 950 & 50,7 & $46,2-55,2$ \\
\hline Fazendo uso de mamadeira & 983 & 61,4 & $55,7-66,3$ & 946 & 77,6 & $70,2-84,3$ \\
\hline
\end{tabular}


valência de amamentação exclusiva, em menores de quatro meses permanece aquém das recomendações da OMS/UNICEF (UNICEF, 1990; WHO, 1994) e da política brasileira, que preconizam a amamentação exclusiva para todas as crianças até 4-6 meses de idade e a introdução de alimentos complementares para todas as crianças de seis a nove meses de idade (INAN/ UNICEF, 1995).

Os resultados obtidos em João Pessoa e em Florianópolis sugerem a existência de processos diferenciados de práticas de alimentação infantil nas cidades em estudo. Todos os indicadores testados demonstram uma melhor situação em Florianópolis, quando comparada com João Pessoa, notadamente em menores de quatro meses. Com uma duração mediana de amamentação de quase oito meses e uma prevalência de amamentação exclusiva, em menores de quatro meses, de 46,3\%, Florianópolis é uma das cidades do país com mais altos níveis de indicadores. João Pessoa apresenta uma situação inversa, com uma duração mediana de amamentação de seis meses e uma prevalência de amamentação exclusiva de 23,9\% em menores de quatro meses.

Os níveis das prevalências em diferentes categorias de alimentação infantil e a duração de cada modalidade de amamentação observados não são satisfatórios. Vários fatores podem estar favorecendo a inadequação de práticas de alimentação dentre os quais pode se pensar no nível de organização dos serviços de saúde e no nível de intensidade das intervenções nas comunidades. Dados não mostrados, obtidos por análise da tabela de contingência, empregando o Epi Info e comparando a prevalência de cada categoria de amamentação obtida em Florianópolis com a observada em João Pessoa, sugerem maior chance de a criança de Florianópolis ser amamentada, em todas as modalidades, do que a de João Pessoa. A razão de prevalência de amamentação exclusiva resultante, em menores de quatro meses, foi de 1,92 (IC 95\% = 1,51-2,45; p = 0,000), favorecendo Florianópolis, quando comparada com João Pessoa. Para as outras modalidades, a razão de prevalência, continuando favorável a Florianópolis, foi de 1,57 (IC 95\% = 1,33-1,85; p = 0,000) com relação à amamentação exclusiva e predominante e de 1,17 (IC 95\% = 1,08-1,27; p = 0,002) no que se refere à amamentação no geral.

Resultados de análise de regressão logística (dados não mostrados) indicam persistência de associação entre cidades (Florianópolis/ João Pessoa) e freqüência de diferentes modalidades de amamentação mesmo quando se controlou o efeito de possíveis variáveis de
Tabela 3

Duração mediana de amamentação (em dias) nas cidades de João Pessoa

e Florianópolis (1997)

\begin{tabular}{lcc}
\hline Modalidade & Florianópolis & João Pessoa \\
\hline Amamentação exclusiva & 53,3 & 16,5 \\
Amamentação exclusiva e predominante & 93,9 & 52,8 \\
Amamentação & 238,4 & 194,8 \\
\hline
\end{tabular}

confusão (idade, paridade, ocupação e escolaridade maternas). A diferença observada entre as duas cidades deve-se, provavelmente, aos atributos maternos e às políticas de alimentação infantil implementadas. Aspectos culturais constituem outro conjunto de fatores que deve ser considerado para entender melhor a relação entre amamentação e local de residência.

As condições econômicas, a idade e a ocupação maternas assim como as rotinas hospitalares fazem parte dos fatores mais citados na literatura especializada (Pérez-Escamilla, 1993; Van Esterik, 1996). Estudos têm revelado que o trabalho fora do lar é um fator de risco para o desmame precoce (Al-Ayed \& Qureshi, 1998). Em São Paulo, observou-se que a licença-maternidade tem sido útil e usada pela maioria das trabalhadoras para amamentar; todavia, outros fatores fundamentais podem interferir negativamente no início e na manutenção da lactação (Rea et al., 1997, 1999). Sabe-se também que o acesso aos serviços de saúde é um potencial fator de confusão na relação entre amamentação e trabalho materno fora do lar (Sanghvi, 1996; Van Esterik, 1996; Rea et al., 1997).

No momento da coleta de dados, cada uma das duas cidades estudadas contava com dois Hospitais Amigos da Criança e com pelo menos um Banco de Leite Humano. Várias atividades de promoção do aleitamento materno já eram desenvolvidas, especialmente, durante a Semana Mundial de Amamentação. Florianópolis parecia mais avançada na organização e no volume das intervenções, havendo um número maior de profissionais capacitados. Nessa cidade, as 48 Unidades Básicas de Saúde (UBS) existentes encontravam-se já comprometidas com a amamentação, e uma delas já tinha adequado à assistência ambulatorial os Dez Passos para o Sucesso do Aleitamento Materno preconizados para os hospitais. Nos últimos anos, a Norma Brasileira de Comercialização de Alimentos para Lactentes (Brasil, 1992) vem sendo objeto de monitoramento, em Florianópolis, enquadrando-se nas ações 
conjuntas da International Baby Food Action Network (IBFAN) e diferentes setores como a Coordenadoria de Proteção e Defesa do Consumidor (PROCON-SC) e a Vigilância Sanitária Municipal.

Sabe-se que em cidades com melhores indicadores de amamentação, como é o caso de Florianópolis, têm sido desenvolvidos intensamente programas de ação com atividades de promoção, apoio e proteção à amamentação. Em cidades como João Pessoa, apesar do desempenho dos profissionais de saúde, nota-se a existência de maior número de problemas na execução das estratégias, sobretudo, no que se refere à disponibilidade de recursos financei-

\section{Agradecimentos}

Os autores agradecem o apoio financeiro do Instituto Nacional de Alimentação e Nutrição/Ministério da Saúde, convênio - 28/96.

\section{Referências}

AL-AYED, I. H. \& QURESHI, I., 1998. Breastfeeding practices in urban Riyadh. Journal of Tropical Pediatrics, 44:113-117.

ARCOVERDE, T. L.; CORREA, D. J. \& MARTINS, J. T.,1997. Perfil do aleitamento materno em uma unidade de saúde do município de Blumenau. In: V Encontro Nacional de Aleitamento Materno, Anais, p. 67. Londrina: Centro de Referência em Amamentação de Londrina/Comitê de Estímulo ao Aleitamento Materno de Londrina/Associação Médica de Londrina/International Baby Food Action Network Brasil.

BARROS, F. C. \& VICTORA, C. G., 1991. Amamentação e desmame. In: Epidemiologia da Saúde Infantil: Um Manual para Diagnósticos Comunitários (F. C. Barros \& C. G. Victora, org.), pp. 32-35, São Paulo: Editora Hucitec/Fundo das Nações Unidas para a Infância.

BEMFAM (Sociedade Civil Bem-Estar da Família no Brasil), 1997. Pesquisa Nacional sobre Demografia $e$ Saúde. Rio de Janeiro: Fundo das Nações Unidas para a Infância.

BOERMA, J. T.; RUTSTEIN, S. O.; SOMMERFELT, A. E. \& BICEGO, G. T., 1991. Bottle use for infant feeding in developing countries: Data from the DHS. Has the bottle been lost? Journal of Tropical Pediatrics, 37:116-120.

BRASIL, 1992. Resolução 31/92. Altera a Resolução 5/88 que Aprovou a Norma Brasileira de Comercialização de Alimentos para Lactentes Incluindo Item sobre o uso de Bicos e Mamadeiras. Brasília: Conselho Nacional de Saúde. ros e de profissionais capacitados para implementação e manutenção das intervenções.

A realização de diagnóstico rápido da situação de amamentação constitui um importante passo para a definição e o redirecionamento de políticas na área de saúde materno-infantil e de nutrição. Espera-se que os resultados deste trabalho sejam aproveitados, para que as cidades possam monitorar a evolução dos indicadores e assim planejar ou redirecionar as intervenções de forma conseqüente. O uso de método rápido e padronizado mostra-se fundamental para qualquer sistema de vigilância em saúde, facilitando a comparabilidade de resultados.
DA SILVA-SIMÕES, C. C. \& MONTEIRO, C. A., 1985. Tendência secular e diferenciais regionais da mortalidade infantil no Brasil. In: Velhos e Novos Males da Saúde no Brasil: A Evolução do País e de suas Doenças (C. A. Monteiro, org.), pp. 153-172, São Paulo: Editora Hucitec/Núcleo de Pesquisas Epidemiológicas em Nutrição e Saúde, Universidade de São Paulo.

DEAN, A. G.; DEAN, J. A.; COULOMBIER, D.; BRENDEL, K. A.; SMITH, D. C.; BURTON, A. H.; DICKER, R. C.; SULLIVAN, K.; FAGAN, R. F. \& ARNER, T. G., 1994. Epi Info, Version 6: A Word Processing Database, and Statistics Program for Epidemiology on Microcomputers. Atlanta: Centers of Disease Control and Prevention.

DE MOURA, E.; CÉSAR-CAMARGO, M.; NETO, A. V. F.; DE CASTRO, C. M. \& ALBUQUERQUE, C. S., 1998. Prevalência da amamentação em Campinas. In: IV Congresso Brasileiro de Epidemiologia, Resumos, p. 26. Rio de Janeiro: ABRASCO.

DE SOUSA, F. J. P., 1992. Pobreza, Desnutrição e Mortalidade Infantil: Condicionantes Sócio-Econômicos. Fortaleza: Fundo das Nações Unidas para a Infância.

DUFFAU, T. G., 1997. Intervalo de confianza. Revista Chilena de Pediatría, 68:239-243.

GARDNER, M. J. \& ALTMAN, D. G., 1986. Confidence intervals rather than $P$ values: Estimation rather hypothesis testing. BMJ, 292:746-750.

INAN (Instituto Nacional de Alimentação e Nutrição)/UNICEF (Fundo das Nações Unidas para a Infância), 1995. Aleitamento Materno e o Municí- 
pio. Rio de Janeiro: Editora Coronário.

LAVINAS, L. \& MAGINA, M., 1996. Atlas Regional das Desigualdades. Banco de Dados com Indicadores Sócio-Econômicos. Rio de Janeiro: Diretoria de Pesquisa, Instituto de Pesquisa Econômica e Aplicada.

MENDES-GOMES, M. A. S.; CAMPOS, T. P.; DE CASTRO, I. R. R. \& BRASIL, C. L. P., 1998. Diagnóstico da situação do aleitamento materno em campanha de vacinação: A experiência do Município do Rio de Janeiro em 1996. In: IV Congresso Brasileiro de Epidemiologia, Resumos, p. 309. Rio de Janeiro: ABRASCO.

MONEGO, E. T.; SILVA, B. H. A. B.; OLIVEIRA, A.; PINTO, S. L.; VENANCIO, S. I. \& KITOKO, P., 1998. Prevalência de aleitamento materno no Estado de Goiás. In: IV Congresso Brasileiro de Epidemiologia, Resumos, p. 311. Rio de Janeiro: ABRASCO.

MONTEIRO, C. A., 1997. O Panorama da Nutrição Infantil nos Anos 90. Brasília: Fundo das Nações Unidas para a Infância.

NORUSIS, M. J., 1993. SPSS for Windows Advanced Statistics, Release 6.0. New York: Prentice-Hall.

NUNES-DA-SILVA, N., 1998. Amostragem por conglomerados. In: Amostragem Probabilística: Um Curso Introdutório (N. Nunes-da-Silva, org.), pp. 75-94, São Paulo: Edusp.

OPS (Organización Panamericana de la Salud)/OMS (Organización Mundial de la Salud), 1991. Indicadores para Evaluar las Prácticas de Lactancia Materna. Washington, D.C.: Centro de Estudio y Documentación, Organización Mundial de la Salud.

PARADA, C. M. G. L.; CARVALHAES, M. A. B. L.; MANOEL, C. M. \& VENANCIO, S. I., 1997. Diagnóstico da situação do aleitamento materno no Município de Botucatu-SP: Utilização de metodologia simplificada. In: V Encontro Nacional de Aleitamento Materno, Anais, p. 75. Londrina: Centro de Referência em Amamentação de Londrina/Comitê de estímulo ao Aleitamento Materno de Londrina/Associação Médica de Londrina/International Baby Food Action Network Brasil.

PEREZ-ESCAMILLA, R., 1993. Patrones de la lactancia natural en América Latina y el Caribe. Boletín de la Oficina Sanitaria Panamericana, 115:185-193.

REA, M. \& BERQUÓ, E., 1995. The programme to promote breast feeding in Brazil: An example of social mobilisation. In: Evaluation of the Impact of Health Interventions (H. Rashad, R. Gray, \& T. Boerma, eds.), pp. 455-472, Liège: International Union for the Scientific Study of Population.

REA, M. F.; VENANCIO, S. I.; BAPTISTA, L. E.; DOS SANTOS, R. G. \& GREINER, T., 1997. Possibilidades e limitações da amamentação entre mulheres trabalhadoras formais. Revista de Saúde Pública, 31:149-156.
REA, M. F; VENANCIO, S. I.; BAPTISTA, L. E.; GREINER, T., 1999. Determinants of the breastfeeding pattern among working women in São Paulo. Journal of Human Lactation, 15:235-239.

SANGHVI, T. G., 1996. Melhora da Eficácia em Função dos Custos da Promoção do Aleitamento Materno em Maternidades. Brasília: Fundo das Nações Unidas para a Infância.

SILVA-AYÇAGUER, L. C., 1995. Excursión a la Regresión Logística en Ciencias de la Salud. Madrid: Ed. Diaz de Santos.

SILVA, L. P.; BARATTA, T. C. \& DA SILVA, M. M., 1991. Como Organizar a Vacinação no município. Rio de Janeiro; Instituto Brasileiro de Administração Municipal/Fundo das Nações Unidas para a Infância. (mimeo.)

UNICEF (Fundo das Nações Unidas para a Infância), 1990. Declaração de Innocenti sobre a Proteção, Promoção e Apoio ao Aleitamento Materno. New York: UNICEF.

UNICEF/SSB (Fundo das Nações Unidas para a Infância/Secretaria de Saúde da Bahia), 1995. Indicadores do Aleitamento Materno: Uma Proposta. Salvador: UNICEF/SSB. (mimeo.)

VAN ESTERIK, P., 1996. The cultural context of breastfeeding and breastfeeding policy. Food and $\mathrm{Nu}$ trition Bulletin, 17:422-30.

VENANCIO, S. I. \& MONTEIRO, C. A., 1998. A tendência da prática da amamentação no Brasil nas décadas de 70 e 80. Revista Brasileira de Epidemiologia, 1:40-49.

VICTORA, C. G.; SMITH, P. G.; VAUGHAN, J. P.; NOBRE, L. C.; LOMBARDI, C.; TEIXEIRA, A. M. B.; FUCHS, S. M.; MOREIRA, L. B.; GIGANTE, L. P. \& BARROS, F. C., 1987. Evidence for protection by breast-feeding against infant deaths infectious diseases in Brazil. Lancet, 8554:319-321.

VIEIRA, G. O.; GLISSER, M.; ARAÚJO, S. P. T. \& SALES A. N., 1998. Indicadores do aleitamento materno na cidade de Feira de Santana, Bahia. Jornal de Pediatria, 74:11-16.

WHO (World Health Organization), 1994. World Health Assembly. Resolution 47.5. Geneva: WHO.

WHO (World Health Organization), 1997. Indicators for Assessing Breastfeeding Practices. Geneva: WHO.

WHO (World Health Organization)/UNICEF (United Nations International Children's Emergency Fund)/USAID (United States of America Agency International Development)/SIDA (Sweden International Development Agency), 1990. Innocenti Declaration on the Protection, Promotion and Support of Breastfeeding. Florence: WHO/UNICEF. 